\title{
Performance degradation and altered cerebral activation during dual performance: Evidence for a bottom-up attentional system
}

\author{
Yunglin Gazes ${ }^{a}$, Brian C. Rakitin ${ }^{a}$, Jason Steffenera ${ }^{a}$ Christian Habeck ${ }^{a}$, Brady Butterfield ${ }^{a}$, \\ Claude Ghez ${ }^{\mathrm{b}}$, and Yaakov Stern ${ }^{\mathrm{a},{ }^{*}}$ \\ ${ }^{a}$ Cognitive Neuroscience Division, Taub Institute, Columbia University, 630 West 168th Street, P \\ \& S Box 16, New York, NY 10032, USA \\ ${ }^{b}$ Department of Neurology and Neuroscience, Columbia University, 821 Psychiatric Institute \\ Annex, 1051 Riverside Drive, New York, NY 10032, USA
}

\begin{abstract}
Subjects performed a continuous tracking concurrently with an intermittent visual detection task to investigate the existence of competition for a capacity-limited stage (a bottleneck stage). Both perceptual and response-related processes between the two tasks were examined behaviorally and the changes in brain activity during dual-tasking relative to single-task were also assessed. Tracking error and joystick speed were analyzed for changes that were time-locked to visual detection stimuli. The associated brain activations were examined with functional magnetic resonance imaging (fMRI). These were analyzed using mixed block and event-related models to tease apart sustained neural activity and activations associated with individual events. Increased tracking error and decreased joystick speed were observed relative to the target stimuli in the dualtask condition only, which supports the existence of a bottleneck stage in response-related processes. Neuroimaging data show decreased activation to target relative to non-target stimuli in the dual-task condition in the left primary motor and somatosensory cortices controlling righthand tracking, consistent with the tracking interference observed in behavioral data. Furthermore, the ventral attention system, rather than the dorsal attention system, was found to mediate task coordination between tracking and visual detection.
\end{abstract}

\section{Keywords}

fMRI; Compensatory tracking; Visual detection; Bottom-up attention system; Psychological refractory period; Dual-task interference

\section{Introduction}

\begin{abstract}
Skilled performance typically requires executing multiple tasks simultaneously, many of which incorporate a continuous task in tandem with discrete tasks. For example, in driving, steering a vehicle is performed simultaneously with acceleration or braking. A consistent finding in dual-task studies, where subjects simultaneously perform two tasks, is the degradation of performance in one or both tasks relative to that observed for the single-task alone $[4,15,21,24,28,34,43,45]$. Understanding the behavioral and the neural mechanisms underlying this degradation is essential to the safe performance of these skills, an important factor especially during vehicle operation. While numerous studies have examined dualtasking with two overlapping discrete tasks behaviorally and with neuroimaging
\end{abstract}

(c) 2010 Elsevier B.V. All rights reserved.

"Corresponding author. Tel.: +1 212342 1350; fax: +1 212342 1838. ys11@ columbia.edu (Y. Stern). 
$[8,15,28,34,39,41,44]$, studies combining behavioral with neuroimaging analyses to understand the effect of a continuous task simultaneously performed with a discrete task are still lacking. There is evidence that continuous tasks are divided into a series of discrete tasks, the scheduling of which may differ from executing discrete tasks alone [34,47]. In well practiced subjects, van Mier et al. [47] found that motor execution for a previous move and motor planning for the next move overlapped in a continuous motor task. In continuous dual-tasking, the amount of interference was found to be dependent on the identity of the overlapping processing stages and was reduced when the retention stage of one task overlapped with the response stage of a second task relative to the interference during overlapping response stage in both tasks [17]. Thus, when a continuous task is performed simultaneously with a discrete task, the mechanism of dual-task interference may be distinct from that found in dual discrete tasks given the flexibility in task scheduling available to continuous tasks. The present study examined dual-tasking with a continuous task, compensatory tracking, and an intermittent task, visual detection, both behaviorally and with functional magnetic resonance imaging (fMRI) to determine the neural changes involved in dual-task interference.

\subsection{Behavioral dual-task studies}

Studies that have tested the effect of a simultaneous discrete task on continuous tracking have found performance decrement in one or both of tracking and the additional task [35]. For example, Griew [13] examined the effect of tracking concurrently with an auditory reaction time (RT) task. Subjects tracked with their left hand while responding to auditory signals with their right hand. Griew found a significant decrease in tracking performance as well as increases in RT relative to the corresponding measures in the single-task condition. Monty and Ruby [30] also found performance decrements in both tasks when tracking was executed simultaneously with a visual motor task. Netick and Klapp [31] reported changes in tracking behavior when concurrently performed with an auditory go/no-go task. Overall, tracking performance was consistently found to be degraded in the dual-task condition.

The sensorimotor processing in many motor tasks is understood to involve serial processing stages from initial perceptual states to final stages concerned with response programming and execution [29,42]. For most tasks, the perceptual stages provide for stimulus detection and identification, and the response stages include selection and execution of the appropriate motor response. Performance degradation is attributed to limitation in the capacity of one or more stages shared between concurrent tasks, causing a bottleneck in the processing stream $[8,34]$. Processing bottlenecks have been found for both perceptual [20] and response stages $[32,34]$. Behaviorally, our study aimed to tease apart perceptual and response processes as the cause of performance degradation.

Both perceptual and response stages may be shared by the two tasks used in our study, compensatory tracking and visual detection, and might cause performance degradation [8]. In the current experimental design, visual stimuli for tracking were confined to the center of the screen while the detection stimuli resided in the periphery. Thus, saccades made to a peripheral detection stimulus from the central tracking field may be detrimental to tracking performance [9]. Deubel and Schneider [9] reported evidence for saccade to be indissociable from visual attention. In our study, saccade made to the detection stimuli may interfere with perception of the tracking cursor movement, and thus may underlie apparent competition for visual processing resources. As regards response processing, even though tracking was executed by the right hand and response to visual detection was made with the left, a common process may be responsible for generating motor instructions for both hands such that while instructions for one hand is processed, the other hand has to halt its movements until processing is completed for the first task. 
In order to identify the bottleneck stage(s) shared by two concurrent tasks, previous studies manipulated different aspects of tasks, such as the stimulus onset asynchrony or the level of task difficulty, and measured changes in RT for both tasks [8,21,24,33]. RT cannot readily be obtained in a continuous tracking task. To circumvent this difficulty we determined the changes in tracking error and movement speed that were time-locked to stimulus onset in the detection task. These variables quantify tracking behavior directly in terms of task performance and motor output, respectively. Thus, processing stages that experience dualtask interference can be identified by comparing trials that require specific stages of stimulus or response processing to ones that do not.

To control for stimulus processing required for the detection task, those same stimuli were also presented in the single-task tracking condition but subjects were told to ignore them. This manipulation maintained consistent visual stimulation between the single and the dualtask conditions and thus the main difference in processing stages between the non-targets in the two conditions was higher order perceptual processes. Increases in tracking error following non-target stimulus onset in the dual but not in the single-task condition would indicate a bottleneck in perceptual processing stages.

Response processing bottleneck would be revealed by changes in tracking error relative to the targets but that would not be observed for the non-target in the dual-task condition. Response-related processes were only active in target stimuli. Therefore, any changes associated only with target stimuli would reflect interference from response processes. If both perceptual and response processes contain processing bottleneck stages, then tracking error and joystick speed should show two periods of change during target stimuli and one period of change during non-target stimuli in the dual-task condition.

\subsection{Neuroimaging dual-task studies}

Neuroimaging studies of dual-tasking have reported two main types of effects. First, reduced dual-task activation relative to single-task regions were reported [12,22,23]. For example, Just et al. [23] compared brain activation in a driving simulation task when performed alone and while responding to true or false verbal questions. The authors reported decreased activation in the parietal lobe while performing both tasks relative to driving-only activation. The deactivation was likely due to interference from the verbal questions, reducing the amount of processing devoted to driving. Even though the interaction between tracking and visual detection in our study may cause brain activation changes different from that between driving and verbal processes, areas associated with tracking, such as the left primary motor and somatosensory cortices, should be affected similarly by a secondary task (i.e. decreased activation relative to single-task tracking).

The second effect seen in neuroimaging studies of dual-tasking is increased activation associated with the scheduling of two conflicting tasks [15,39-41,44]. Task order control is the assignment of priority to two simultaneous tasks for access to a bottleneck process. It becomes essential when conflict arises between two tasks and requires proportioning attention to the more immediate task, thus providing the task with access priority to the bottleneck stage. To locate brain regions associated with task order control, trials that need active task order control can be compared with those that only need passive control, such as task switching trials (active reversal of task order) relative to non-switch trials (same task order as previous trial) [44]. Any region that shows greater activation in the switch trials than the non-switch trials would be involved in task order control. Both Szameitat et al. [44] and Stelzel et al. [41] reported increased activation in the lateral prefrontal cortex to be associated with task scheduling. Alternatively, the intermittent appearance of detection stimuli may also trigger bottom-up attentional areas [6], drawing attention away from the primary task [27]. The bottom-up attention system differs from task order control in that it is 
externally motivated rather than guided by internal goals, thus it can replace the need for task order control in order to resolve dual-task conflict. Corbetta and Shulman [6] reported activation in the inferior frontal region to be part of the bottom-up attention system whereas the lateral prefrontal cortex is associated with active task scheduling in Szameitat et al. [44]. The pattern of frontal activation associated with a processing bottleneck stage in our result can elucidate the importance of each system in dual-tasking involving a continuous task.

To summarize we hypothesized that a bottleneck stage would produce: (a) increased tracking error time-locked to detection stimulus onset, (b) decreased joystick speed timelocked to detection stimulus onset, (c) decreased brain activation in areas mediating tracking performance, and (d) increased activation in the frontal cortex. Observation of these four effects would provide evidence of a bottleneck stage in the: (a) perceptual stages if the effects are found in the comparison between non-target stimuli in the single vs. the dual-task condition, and (b) response-related stages if the effects are found in dual-task target rather than non-target stimuli.

\section{Methods}

\subsection{Participants}

Two separate groups of subjects were included in the study. Both were tested under the same conditions, but the second group later participated in a sleep deprivation study. Behavioral and fMRI data for the second group were acquired before subjects were sleep deprived. All subjects were right-handed and screened to ensure that they had no history of medical, psychiatric, neurological, sleep disorder, or color-blindness. In group 1, 26 subjects were recruited but 4 had to be exclude (one subject excluded due to MR artifact, one subject due to poor performance- the mean tracking error was at maximum value, and two subjects due to equipment failure), leaving 22 subjects for the analyses. Subjects ranged in age 20-33 years old (mean $=26.4 \pm 3.4$ years). In group 2,34 out of 37 participants were included in the analyses and they ranged in age from 18 to 34 years (mean $=23.1 \pm 3.1$ years). Three subjects were excluded for poor behavioral performance: two subjects did not perform the tracking task and one subject responded to the detection task in the single-task condition. Informed consent, as approved by the Internal Review Board of the College of Physicians and Surgeons of Columbia University, was obtained prior to study participation, and after the nature and risks of the study were explained. Subjects were paid for their participation in the study.

\subsection{Task}

All subjects were tested in both single and dual-task conditions. For the single-task condition they performed only a visuomotor compensatory tracking task. In the dual-task they also performed a visual detection task. In order to match visual stimulation, the visual stimuli for this matching task were also presented during the single-task condition but subjects were instructed to make no response to these cues.

Three phases of the study included titration, training, and testing. For titration and training, subjects sat facing a computer monitor $(30.5 \mathrm{~cm})$. Their right hand held a joystick through which they could control the motions of a cursor, and their left index finger was on the right button of a mouse to respond to the detection task. For testing, subjects lay in the MRI scanner. Task stimuli were back-projected onto a screen located at the foot of the MRI bed using an LCD projector. Stimuli subtended $10.5^{\circ}$ by $10.5^{\circ}$ visual angle on the screen. Subjects viewed the screen via a mirror system located in the head coil. All subjects had vision corrected to as normal as needed using MR compatible glasses (manufactured by Safe Vision, LLC, Webster Groves, MO). Tracking control was performed with an MRcompatible joystick (Resonance Technology) placed to the right of subject's thighs. 
Detection responses were made on a LUMItouch response system (Photon Control Company) with the response paddle placed to the left of subject's thighs. Task onset was electronically synchronized with the MRI acquisition computer. Task administration and data collection were controlled using REALbasic. A Carnegie Mellon Button Box (New Micros, Inc., Dallas, TX) provided digital input-output for the response system and synchronization with the MRI acquisition computer, as well as millisecond accurate timing of responses.

For the compensatory tracking, a white plus sign constituted the reference position and was drawn at a fixed location at the center of the screen. At trial onset the cursor was aligned with the crosshair. Cursor position was updated every $40 \mathrm{~ms}$. At each update, a continuously varying pseudorandom perturbation was added to the previous $x$ and $y$ coordinates of the cursor. This had the effect of moving the cursor away from the center as though an unpredictable force had been applied to the subject's hand. The task was then to maintain the cursor position aligned with the reference position (the plus sign) and to counteract the perturbation force. The cursor position at each update was further determined by the net sum of all previous joystick movements such that a 2 unit movement in the positive $\times$ direction would continue to push the cursor to the right by 2 units until a joystick movement in the negative $\times$ direction of equal magnitude was made. The maximum cursor displacement from the reference point was 100 pixels at a screen resolution of 800 by 600 pixels.

In order to equalize task difficulty across subjects, perturbation magnitude was titrated for each subject according to their performance in the single-task condition. Titration was standardized to a median tracking error of 15 pixels by adjusting the perturbation magnitude based on performance across thirty 30-s blocks with an inter-trial interval (ITI) of $2 \mathrm{~s}$ for a total of $16 \mathrm{~min}$.

For both training and testing, each tracking trial lasted 2 min. Training for the task lasted 27.4 min on 6 trials each of single and dual-task conditions. Testing in the MRI scanner consisted of 3 blocks in which both conditions were presented twice and randomly ordered across subjects. Each testing block was 11.87 min with a 40-s rest interval and 2-s instruction screen at the start of each tracking trial. The rest screen presented the same visual stimuli as in a test trial instead of a non-blank rest screen in order to subtract visual activation from all functional activations.

For the visual detection task, stimuli consisted of four circles arranged one in each of the four corners of the screen appearing concurrently with the tracking cursor. The circles start with one of the four possible colors (red, blue, green, and yellow), and may take on any of the four possible colors every few seconds. Color match between diagonally positioned circles constituted a target signal and subjects were instructed to respond with a button press using their left index finger, while any other circle color change was a non-target stimulus. Six target stimuli were presented in every 2-min tracking trial. Mean ITI for target stimuli was $19.3 \pm 10.0 \mathrm{~s}$, and each target stimulus remained on the screen for $8 \mathrm{~s}$. Mean ITI for non-target stimuli was $3.86 \pm 2.44 \mathrm{~s}$ with a range of $2-12 \mathrm{~s}$. To maintain equal visual stimulation between the two conditions, detection stimuli were presented in the single-task condition, but subjects were instructed to ignore the detection stimulus. Target and nontarget stimuli were presented at the same frequency as in the dual-task condition. A $2 \mathrm{~s}$ instruction screen before each tracking trial informed subjects of the condition type to be performed. The word 'Track' remained on the left and right side of the screen throughout single-task trials and 'Circles' in dual-task trials. 


\subsection{Procedure}

Before testing, subjects were trained to perform the tasks and perturbations were titrated. For group 1, titration and training were performed on the same day as testing whereas group 2 subjects were titrated and trained two weeks prior to testing in accordance with the protocol of the larger study. For tracking, the behavioral data included tracking error, joystick position, cursor position, perturbation, and for visual detection, the onsets of target and nontarget stimuli, and RT for target responses.

\section{4. fMRI data acquisition}

During each block of testing, $285 \mathrm{~T} 2 *$-weighted images (BOLD images) [28], were acquired with an Intera 1.5-T Phillips MR scanner equipped with a 6-channel SENSE head coil [36], using a gradient echo echo-planar (GE-EPI) sequence (time echo/time repetition [TE/TR] = $50 / 2500 \mathrm{~ms}$; flip angle $=90^{\circ} ; 80 \times 80$ matrix, in-plane voxel size $=3.124 \mathrm{~mm} \times 3.124 \mathrm{~mm}$; slice thickness $=6 \mathrm{~mm}$ [no gap]; 25 trans-axial slices per volume; interleaved slice acquisition). Four additional GE-EPI excitations were performed before the task began to allow transverse magnetization to approach its steady-state value; the data for these excitations were discarded. A T1-weighted spoiled gradient image was acquired from each subject for spatial normalization purposes (TE/TR $=3 / 25 \mathrm{~ms}$; flip angle $=45^{\circ}, 256 \times 256$ matrix; in-plane voxel size $=0.781 \mathrm{~mm} \times 0.781 \mathrm{~mm}$; slice thickness $=1.5 \mathrm{~mm}$ [no gap]; 124 trans-axial slices per volume).

\subsection{Behavioral data analysis}

2.5.1. Tracking error-Root-mean-square (RMS) of the tracking error was calculated using the formula $\sqrt{\operatorname{mean}\left(x^{2}+y^{2}\right)}$, where $x$ and $y$ were the horizontal and vertical cursor distances at each time point, respectively, from the center of the screen in pixels. Screen size was approximately 82 pixels per inch.

2.5.2. Joystick speed—Joystick speed between two time points $\left(t_{1}, t_{2}\right)$ was calculated by

$$
\frac{\sqrt{\left(j_{x 2}-j_{x 1}\right)^{2}+\left(j_{y 2}-j_{y 1}\right)^{2}}}{t_{2}-t_{1}}
$$

with $j_{X}$ and $j_{y}$ being the joystick's horizontal and vertical distance, respectively, from the joystick's neutral position. Joystick speed was then low-pass filtered with 101 order finite impulse response filter having a cut-off frequency of $0.2 \mathrm{~Hz}$. The order and cut-off frequency were selected to filter out high frequency changes while preserving the trend in joystick movement. Low-pass filtering joystick speed allowed investigation of the slower metabolic change in the brain in relation to movement.

2.5.3. Trial means analyses-Repeated-measures ANOVAs were conducted separately for tracking error, joystick speed, reaction time to detection stimuli, the number of hits (correct response to target trials), and the number of false alarms. Each ANOVA consisted of 2 conditions (single/dual-task) $\times 3$ blocks $\times 2$ trials with group $(1$ and 2$)$ as the betweensubjects factor. $P$-values for factors that did not satisfy the sphericity assumption were corrected using the Huynh-Feldt correction.

2.5.4. Binned time-series analyses-To explore temporal changes in tracking that might not be detectable in the mean values for each trial, tracking error and joystick speed 
were binned and time-locked to detection stimulus onset. The presence of a bottleneck stage would be shown by comparisons between: (1) non-target stimuli in the single vs. dual-task condition for perceptual stages and (2) target vs. non-target stimuli in the dual-task condition for response stages.

Stimulus-locked tracking error and joystick speed were each divided into $640 \mathrm{~ms}$ bins from $8000 \mathrm{~ms}$ before to $8000 \mathrm{~ms}$ after onset for each detection stimulus type. This interval was chosen because $8000 \mathrm{~ms}$ was the duration of each target stimulus presentation during which only one target stimulus was on the screen. Bin width of $640 \mathrm{~ms}$ divided the $16,000 \mathrm{~ms}$ window of interest into 25 bins. For both variables, binned time-series data were calculated separately for target and non-target stimuli within both single and dual-task conditions. Nontarget stimuli only included circle color changes that did not overlap with a target stimulus. Tracking error and joystick speed time-series were each analyzed using an ANOVA design consisting of 2 conditions $\times 2$ stimulus types $\times 25$ bins $\times 2$ groups. A priori contrast analyses were performed by comparing each bin mean to the grand mean of the 25 bins.

\section{6. $\mathrm{fMRI}$ statistical analysis}

2.6.1. Preprocessing-All image preprocessing and analysis were implemented using the SPM5 software package (Wellcome Department of Cognitive Neurology [10]) and other in-house code written in MATLAB 7.1 (Mathworks, Natick, MA). The following procedures were used for each subject's GE-EPI data set. Data were temporally shifted to correct for the order of slice acquisition, using the first slice acquired in the TR as the reference. All GE-EPI images were realigned to the first volume of the first session and then coregistered to the T1-weighted (structural) image using mutual information. The structural image was then used to determine parameters for transformation into standard space defined by the Montreal Neurologic Institute template brain supplied with SPM5. This transformation was then applied to the GE-EPI data, which were resliced using sincinterpolation to $2 \mathrm{~mm} \times 2 \mathrm{~mm} \times 2 \mathrm{~mm}$ and then spatially smoothed with an $8 \mathrm{~mm}$ fullwidth-at-half-maximum (FWHM) kernel.

2.6.2. Time-series modeling-The fMRI data analysis comprised two levels of voxelwise general linear model (GLM) [16]: the first-level GLM is a time-series analysis that yields summary measures to be used in the second-level GLM, which affords statistical inference at the group level. In the (subject-separable) first-level GLM, the GE-EPI timeseries were modeled with regressors representing the expected BOLD fMRI response to each condition (single and dual-task) and to target and non-target stimuli in each condition for each of the three blocks plus a regressor representing the instruction screen for each block. Additional regressors were added for false alarms and misses, which varied by block and subject. For each block, both single and dual-task condition regressors were parametrically modulated by the corresponding joystick speed to locate brain regions that correlated with joystick speed across time. The regressors were constructed by convolutions of an indicator sequence (i.e. a train of discrete-time delta functions) representing tracking and detection event onsets, an assumed BOLD impulse response function (as represented by default in SPM5), and a rectangular function of duration dictated by the duration of the assumed neural response [49]. In particular, correctly responded target stimulus regressors were modeled with event durations equaling to the respective reaction time [14].

In order to minimize the correlation between predictors in the time-series model, the nontarget onset predictors were orthogonalized with respect to the tracking predictor. The tracking predictor contained the mean activation of the entire trial, which included not only activity related to tracking, but also any activity that persisted for most of the trial. Since non-target stimuli occurred fairly frequently throughout the trial, it was unavoidable that the 
tracking block contained some variability associated with non-target stimuli. For the nontarget predictor, orthogonalization only removed the mean from the predictor, but the predictor still corresponded to the time-varying signal change associated with non-target event onsets, enabling valid interpretation of this predictor after orthogonalization. The contrast estimate images were then used as the dependent variable in a second-level GLM.

2.6.3. Group analyses-Group GLM was performed on four effects of interest: (a) baseline activation associated with visuomotor tracking, (b) dual vs. single-task tracking, (c) non-target stimuli in the single vs. the dual-task condition, and (d) target vs. non-target stimuli in the dual-task condition. For each model, univariate statistical parametric modeling [SPM: [11]] was performed to localize regions showing the effects of interest. Contrasts of the parameter estimates from single-subject models were entered into random-effects analyses (repeated-measures ANOVA). Group identity was entered as the between-group factor in each model. Except where mentioned, results were manually corrected for multiple comparisons across the whole-brain using the Bonferroni correction for the number of resolution elements [48], using an extent threshold of 10 contiguous voxels. Likely cytoarchitectonic labels for cluster maxima in these thresholded patterns were obtained using Talairach daemon software [26] and checked with visual inspection.

\section{Results}

\subsection{Behavioral data}

Behavioral findings were analyzed in terms of trial means and of the means of binned data time-locked to detection stimulus onset. Trial means ANOVA tested the effects of Condition $\times$ Block $\times$ Trial $\times$ Group on the dependent variables tracking error, joystick speed, and false alarms, and for the dependent variables RT to target response and the number of correct responses, the effects were Block $\times$ Trial $\times$ Group. Binned means ANOVA for stimuluslocked tracking error and joystick speed contained the factors Condition $\times$ Stimulus type $\times$ Bin $\times$ Group. The trials means analyses are presented first, followed by the binned means analyses, then group effects are discussed.

\subsubsection{Trial means analyses}

3.1.1.1. Tracking variables: The mean tracking error across both conditions was $22.89 \pm$ 0.938 pixels. Increased tracking error was observed in the dual-task condition relative to the single-task condition; the dual-task condition was more difficult than the single-task condition, $F(1,50)=4.262, p<0.05$. Significant interaction between Block and Condition showed that tracking error remained constant across blocks in the single-task condition but in the dual-task condition tracking error decreased from blocks 1 to $3, F(2,100)=3.728, p<$ 0.05 , which may be attributed to a learning effect in the more difficult condition. The main effects Block and Trial were not significant (Block: $F(2,100)=0.905, p>0.05$; Trial: $F(1,50)=2.458, p>0.05)$, but their interaction reached significance, $F(2,100)=4.069, p<$ 0.05 . From trial 1 to 2, block 1 error decreased across the trials, but in blocks 2 and 3 error increased instead. Mean joystick speed was $7.305 \pm 0.564$ pixels per second. Joystick speed was similar in the two conditions, $F(1,50)=0.148, p>0.05$, but it increased across blocks, $F(2,100)=5.492, p<0.05$. Trial and Group main effects and their interactions did not reach significance (Trial: $F(1,50)=3.512, p>0.05$; Group: $F(1,50)=0.189, p>0.05$ ).

3.1.1.2. Visual detection variables: Subjects performed the detection task with minimal error; the overall mean number of hits was $5.643 \pm 0.051$ out of 6 target stimuli presented per tracking trial. None of the effects tested in the hits analysis reached significance (Block: $F(2,100)=1.481, p>0.05$; Trial: $F(1,50)=2.286, p>0.05$; Group: $F(1,50)=1.031, p>$ $0.05)$. Mean RT to target stimuli was $1.094 \pm 33.67 \mathrm{~ms}$. Subjects reacted more slowly to the 
detection stimuli as testing proceeded, with RT increasing across blocks, from 1037 to 1120 ms, $F(2,100)=4.809, p<0.05$. Trial and Group effects and their interactions were not significant (Trial: $F(1,50)=1.834, p>0.05$; Group: $F(1,50)=0.361, p>0.05)$. (Since we did not have a single-task visual detection block, we do not know how much longer RT was relative to single-task.) On average, subjects had an average of $0.900 \pm 0.162$ false alarms per tracking trial. There were more false alarms in the dual than in the single-task condition, $F(1,50)=41.69, p<0.001$. The number of false alarms remained low across blocks in the single-task condition but followed a decreasing trend during the dual-task condition from block 1 to $3, F(2,100)=4.459, p<0.05$. The corresponding main effect of block was also significant, $F(2,100)=4.470, p<0.05$. Thus, the number of false alarms showed a small learning effect in the detection task across blocks but the alertness, as measured by RT, deteriorated slightly as testing progressed, but most importantly, accuracy for detection task was near perfect.

3.1.2. Binned means analyses-Here we examine the changes in tracking error and joystick speed relative to onsets of the two detection stimulus types in both test conditions. ANOVA results for both tracking error and joystick speed showed that the bin means were different between conditions and stimulus types (tracking error: $F(24,1296)=3.632, p<$ 0.001 ; joystick speed: $F(24,1296)=5.369, p<0.001)$, with most of the corresponding interactions and main effects also significant. Deviation contrast comparisons located significant changes in the bins after target stimulus presentations in the dual-task condition only. No change was observed in bins relative to non-target stimuli in both single and dualtask conditions as well as in bins relative to target stimuli in the single-task condition. The results suggest that tracking error and joystick speed were not affected by the detection stimuli in the single-task condition or by non-target stimuli in the dual-task condition (see Fig. 1 top), but that tracking error increased and joystick speed decreased significantly after the target stimulus onset in the dual-task condition. The lack of change in tracking variables during non-target stimuli in both conditions is consistent with the lack of a bottleneck stage in perceptual processes. On the other hand, target stimuli induced a significant change in tracking error and joystick speed, indicating a bottleneck stage in response processing.

3.1.3. Group effect-The two groups showed similar effects in task manipulations except for the Block and the Trial effects in tracking error and for the Condition effect in false alarms. Group 2 subjects reduced tracking error with practice, i.e. with successive blocks and trials while group 1's performance remained constant $(F(2,100)=3.727, p<0.05$ for blocks; and $F(1,50)=5.282, p<0.05$ for trials). The decrease may be attributed to the longer time interval between training and testing for group 2 than group 1, resulting in the improvement in performance observed. Group 2 subjects also displayed more false alarms than subjects in group $1, F(1,50)=15.14, p<0.001$. More specifically, the increase in false alarms from the single to the dual-task condition was greater in group 2 than group 1, $F(1,50)=17.18, p<0.001$. Group 2 subjects underwent a separate test in the MRI prior to the tracking test session. The heavier testing load placed on group 2 subjects may have resulted in a higher level of fatigue in these subjects, which may have increased the number of false alarms committed. For the binned time-series analyses, various bins showed significant differences in the deviation contrasts for interactions involving the group factor, but these differences did not show any remarkable trend with respect to stimulus onset. As the bottom of Fig. 1 illustrates, both groups followed the same overall trend relative to stimulus presentation and response for both tracking error and joystick speed. Thus, it is likely that the significant differences found were spurious effects. The overall behavioral result suggests that the two groups displayed similar performance. 


\subsection{Imaging results}

3.2.1. Single-task tracking-Table 1 shows the brain regions activated in this analysis. The $\mathrm{t}$-threshold was corrected with family-wise error correction, at $p<0.05$ and an extent threshold of 50 contiguous voxels. Single-task tracking positively activated the left primary motor cortex, the left premotor cortex, and the left somatosensory cortex consistent with the use of the right hand in tracking. Bilateral supplementary motor area (SMA), bilateral inferior parietal lobule, and bilateral extrastriate cortex including area V5 (MT), and the right cerebellum, in particular the right culmen and the declive (lobules V and VI) were also activated. Tracking was associated with decreased activation relative to baseline in the extrastriate cortex, bilaterally.

3.2.2. Dual vs. single-task tracking-Brain areas associated with greater activation in the dual vs. single-task condition were bilateral extrastriate cortices and left inferior parietal lobule (Table 2). No region showed decreased activation in dual relative to single-task tracking.

3.2.3. Dual vs. single-task non-target stimuli-The goal of comparing brain activity during non-target stimuli in the dual vs. the single-task condition was to identify areas associated with a bottleneck stage in the perceptual processes. Table 3 and Fig. 2 show the activated areas. Regions with greater activity in the dual than single-task condition in this analysis were bilateral extrastriate cortices, right superior parietal lobule, and left inferior frontal gyrus. There was no activation greater in the single than the dual-task condition for non-target stimuli.

3.2.4. Target vs. non-target stimuli in the dual-task condition-Comparison of target and non-target stimuli was intended to reveal brain areas associated with a bottleneck stage in response processing. Table 4 and Fig. 3 show the activated regions in this comparison. Greater activation was found in target than non-target stimuli in the right primary motor cortex, somatosensory cortex, thalamus, bilateral anterior cingulate, inferior frontal gyrus (BA 47), and anterior insula, left inferior parietal cortex, and declive in the left cerebellum. Decreased activation in target than non-target stimuli was found in the left primary motor and somatosensory cortices, consistent with the behavioral finding that a bottleneck stage exists in response processing.

3.2.5. Group differences-There were no group differences for the contrasts single-task tracking and dual vs. single-task tracking, but group differences were found for dual vs. single-task non-target stimuli and dual-task non-target vs. target stimuli. Table 5 shows the neuroanatomical labels for regions with differential activation between the two groups. These significant clusters each consisted of 22 voxels or smaller and were sparsely distributed across the brain, which suggests that these activations were likely due to Type I error rather than true group effects.

\section{Discussion}

The goals of this study were to evaluate whether dual-task interference in continuous tracking results from bottleneck stages in the perceptual and/or response-related processes, and using fMRI to examine changes in brain activation when simultaneously performing a continuous and a discrete task.

\subsection{Perception}

The dual-task effect of perceptual processing was tested with non-target stimuli in the single vs. in the dual-task condition. Since color identification and comparison were not required in 
the single-task condition, visual processing in the single-task condition can be assumed to be limited. Any difference exhibited only during non-target stimuli in the dual-task condition was attributable to greater perceptual load during dual-task trials. The binned time-series analyses did not show any change in tracking error or joystick speed between single and dual-task non-target stimuli, and thus tracking was not interrupted by the additional simultaneous visual processes recruited by the secondary task, supporting the conclusion that visual perception was not a bottleneck stage that interrupts continuous tracking performance. Our result replicates Netick and Klapp's [31] study despite differences in stimulus modality (visual vs. auditory) of the discrete task. However, their study design differed from that in the current study in that they used a precue to signal target instead of a control condition for non-target stimuli, thus precluding an analysis directly comparable to ours. Even with design differences, the lack of performance changes relative to non-target stimuli across time is consistent with our result.

It is also possible that dual-task performance cost during perception only occurred in the visual detection task, thus not observable in tracking performance. Ideally, performance during single-task visual detection would be compared to detection performance during dual-tasking. Being that non-targets do not elicit any response, however, performance change can only be observed in targets, which contain both perceptual and response stages. Thus, perceptual dual-task effect cannot be separated from response effects in our study design, which was intended to examine the effect of dual-tasking on continuous tracking and is not suitable to measure dual-task effect on the discrete task. Future studies should devise a different experimental design that will enable examination of the perceptual dual-task effect on a discrete task due to a concurrent continuous task.

Increased brain activation was found in dual-task non-target relative to non-target in the single-task tracking condition even though behavioral result suggested the lack of a perceptual bottleneck. Given that interference was not apparent in behavior, the associated increased brain activation was likely due to a higher perceptual demand imposed by the additional visual detection task. These regions may have been associated purely with the processing of the detection task. To further investigate this possibility, future studies should include a single-task condition for visual detection and compare the brain activation pattern associated with single-task visual detection to the regions found in the current study.

\subsection{Response processing}

Tracking interference was only observed in target stimuli around the onset of target response and manifested by significant decrements in joystick speed from $200 \mathrm{~ms}$ before to $400 \mathrm{~ms}$ after response followed by significant increase in tracking error at approximately $640 \mathrm{~ms}$ after response. Changes in tracking variables observed only in target stimuli are consistent with a bottleneck stage in response-related processes, which replicated Netick and Klapp's [31] result, in which the researchers reported increased hesitation frequency (a measure of the number of times the tracking hand stopped movement) relative to an auditory go signal while no effect was found in response to a no-go signal.

Since a bottleneck stage is a process shared by both tasks, a likely candidate for a bottleneck stage would be a function that is performed by the same region for both hands. One such process is movement selection, found to be related to activity in the left premotor cortex [37]. Studies using Positron Emission Tomography [38] and Transcranial Magnetic Stimulation [19] reported the involvement of an area in the left premotor cortex in the movement selection of both the left and the right hands. Our imaging results are consistent with the interpretation that the left premotor cortex is a bottleneck in our study design: the left premotor cortex was activated during single-task tracking as well as for dual-task targets (result not reported here), illustrating the involvement of this region in both tasks. While it is 
possible that the dual-task target contrast does not necessarily have to be composed of activation due to the visual detection task and may include tracking activity, based on the observed significant decrease in joystick speed as well as the decreased left primary motor activity during dual-task target response, it is unlikely that tracking activity contributed significantly to the left premotor activation in the dual-task target contrast.

Recent studies reported the phenomenon of crosstalk between conflicting responses in dualtask studies (e.g. left vs. right). Koch [25] showed that left/right button press was delayed when a non-task-related but simultaneous visual-verbal task elicits response in the opposite direction (a right side response when a left button press is the correct response and vice versa). Crosstalk may be a different mechanism from the bottleneck observed in our study because crosstalk is elicited by two opposing responses whereas in our study design the movement performed by the left and the right hands are unrelated to each other: downward button press vs. forward/backward and left/right movements. Furthermore, left premotor cortex is not likely to be involved in crosstalk because crosstalk can occur among any modality (e.g. manual and verbal) suggesting a higher level processing than at the motor processing level.

It was hypothesized that a bottleneck stage should be associated with two effects in brain activation: decreased activation in dual-task relative to single-task in regions associated with tracking, and increased activation in frontal regions associated with attention regulation. Comparison between target and non-target stimuli in the dual-task condition revealed the hypothesized up and down regulation of neural activity. Decreased activation in target relative to non-target stimuli was observed in the left primary motor and the left somatosensory cortex. Our result is consistent with the down regulation of task-related regions during dual-tasking reported by previous dual-task studies [12,22,23]. Our study showed highly significant activation in the left primary motor and the left somatosensory cortex in the single-task condition, but under dual-task load, activity in these regions decreased significantly. These regions are associated with the control of right hand movement; decreased activation in these regions is consistent with exaggerated tracking error and slowed tracking speed concurrent with target responses. Activity in the primary motor cortex was positively correlated with the amplitude of movement in a stepwise tracking task [46]. The decreased activity in the primary motor cortex observed in our study, therefore, likely contributed to decreased joystick speed which then, due to decreased amounts of error correction movement, resulted in increased tracking error. Thus, the down regulation of the primary motor cortex and somatosensory cortex during target responses attributed to the increase in tracking error.

Greater activation in target than non-target stimuli were found in regions associated with left-hand motor response as well as pre-frontal regions known to participate in attention control, which was consistent with the hypothesized increase in brain activation associated with task control. Of the significant regions observed, the anterior insula, the anterior cingulate cortex, the inferior frontal gyrus and the inferior parietal gyrus form the core areas of the ventral attention system [3,6]. Corbetta and Shulman [6] presented evidence for a ventral attention system that was stimulus driven such that stimulus changes activated this system to enable a bottom-up orientation of attention. This is distinguished from a complementary, or top-down mechanism, orienting attention. This latter system is essential for internal control of orientation guided by goals and is embodied in activation of the dorsal attention system [6]. The target stimulus onset most likely triggered the ventral attention system, engaging and re-orienting attention whenever a target stimulus was presented. Activation of the ventral attention system by target stimuli was likely caused by the higher saliency associated with target stimuli due to the lower presentation frequency of targets relative to non-targets - target stimuli being presented only six times per 2-min tracking trial 
while non-targets were presented every few seconds. This is consistent with the lack of activation in our study in the prefrontal cortex (specifically BA 6,9, and 44) as was reported by Szameitat et al. [44] to be involved in task order control. These regions were hypothesized to resolve conflicting dual-task processes. However, the design used by Szameitat et al. was task switching which required goal-motivated attentional orientation towards the first of a pair of tasks. In our task, the higher salience of target stimuli may have obviated the need for active selection of task order, providing the detection task priority access to the bottleneck stage, the premotor cortex in our task, while the tracking task temporarily slowed down. The role of the bottom-up attention system was limited to the detection of targets, after which the more immediate detection task was processed by the premotor cortex first. Since instructions were temporarily lacking for the tracking task, the tracking hand slowed its movement until the premotor cortex completes processing for the detection task and resumes sending tracking instructions. It is also possible, however, that tracking had to be actively inhibited upon detection of the target. Activation of the right inferior frontal cortex has been associated with inhibition of ongoing action [2,5]. The role of the right inferior frontal region in the bottom-up attention system observed in our result may be to suppress any ongoing action as an alarming stimulus is detected.

\subsection{Sustained activation}

Neuroimaging data showed an increase in activation in the right middle occipital region, the left posterior fusiform gyrus, and the left inferior parietal lobule throughout dual-task tracking relative to single-task tracking. The GLM model used in the SPM analysis was able to tease out persistent activations throughout tracking trials from event-related activations. Therefore, the three significant regions were in fact sustained throughout dual-task tracking. Sustained activation in the occipital region could be attributed to the increase in visual processing required for both cursor movements and colored detection stimuli. Left fusiform gyrus was associated with color discrimination [1], which was an important process in visual detection stimulus identification. Activation of visual detection stimulus feature discrimination may have been sustained throughout the tracking trials because there were stimulus presentations every few seconds throughout the trials resulting in constant activation in these regions. Inferior parietal lobule activation was associated with visual attention [7] for which there was increased demand in the dual-task condition.

\subsection{Continuous-discrete vs. discrete-discrete dual-task interference}

Our study simultaneously employed a continuous and a discrete task to study the effect of dual-task interference on the continuous task. Our results suggest differences as well as similarities between interference observed in continuous-discrete and discrete-discrete dualtasks. Perceptual dual-task interference was reported by Jiang [18] in which stimuli presented in the periphery caused longer RT than when stimuli was presented in the center. While our visual detection stimuli were presented in the periphery, the lack of perceptual interference may be attributed to the flexible scheduling between tracking and visual detection which enables the processing of the visual detection stimuli while motor execution is performed for tracking [47], thus avoiding conflicting need for a shared process.

On the other hand, response bottleneck was observed in our study. In discrete-discrete dualtasking, interference during response has been associated with both response selection [34] and motor execution [24]. Herath et al. [15] further localized the right inferior frontal cortex to be involved in dual-task interference between a visual and a somatosensory RT task, which is also a brain region active in our task during dual-task interference between continuous and discrete tasks. Thus, our study suggests dual-tasking between a continuous and a discrete task agrees with the response bottleneck observed between concurrent discrete tasks. A possible reason that interference was not avoided for response stages in our 
continuous-discrete dual-task design is that the length of the visual detection response stages may be longer than the tracking perceptual stages. When a response is made to the target, even though the perceptual stages for tracking may take place simultaneously with response processing for visual detection, tracking perceptual stages are completed earlier than response processing for the detection task, and since response to both tasks share the same process, tracking response processing is delayed until detection response processing is completed.

\section{Conclusion}

Continuous tracking performed concurrently with a visual detection task caused degradation in tracking when response was made to target stimuli, consistent with a bottleneck stage in response processes. Decreased brain activity in the left primary motor cortex likely attributed to worsened tracking performance by decreasing the amount of error-correcting movement. Increased activation in regions associated with the bottom-up attention system suggests that conflict between the two tasks was likely resolved by re-orientation of attention to target stimuli, which was assigned a higher processing priority due to its rare occurrence compared to the continuous tracking task. Our study provided evidence for dualtask conflict resolution by the bottom-up attention system, a system rarely associated with dual-task conflict resolution.

\section{Acknowledgments}

This work was supported by Army Research Office and the Defense Advanced Research Projects Agency for funding support (ARO and DARPA Grant DAAD19-02-1-0047). The views, opinions, and/or findings contained in this article/presentation are those of the author/presenter and should not be interpreted as representing the official views or policies, either expressed or implied, of the Defense Advanced Research Projects Agency or the Department of Defense. We thank Oksana Tatarina-Nulman, Linda Sanders, and Gray Herzberg for their help in data collection.

\section{References}

1. Bartels A, Zeki S. The architecture of the colour centre in the human visual brain: new results and a review. European Journal of Neuroscience. 2000; 12:172-93. [PubMed: 10651872]

2. Blasi G, Goldberg TE, Weickert T, Das S, Kohn P, Zoltick B, et al. Brain regions underlying response inhibition and interference monitoring and suppression. The European Journal of Neuroscience. 2006; 23:1658-64. [PubMed: 16553630]

3. Braver TS, Barch DM, Gray JR, Molfese DL, Snyder A. Anterior cingulate cortex and response conflict: effects of frequency, inhibition and errors. Cerebral Cortex. 2001; 11:825-36. [PubMed: 11532888]

4. Brown SW. Attentional resources in timing: interference effects in concurrent temporal and nontemporal working memory tasks. Perception \& Psychophysics. 1997; 59:1118-40. [PubMed: 9360484]

5. Chikazoe J, Jimura K, Asari T, Yamashita K, Morimoto H, Hirose S, et al. Functional dissociation in right inferior frontal cortex during performance of go/no-go task. Cerebral Cortex. 2009; 19:14652. [PubMed: 18445602]

6. Corbetta M, Shulman GL. Control of goal-directed and stimulus-driven attention in the brain. Nature Reviews Neuroscience. 2002; 3:201-15.

7. Culham JC, Kanwisher NG. Neuroimaging of cognitive functions in human parietal cortex. Current Opinion in Neurobiology. 2001; 11:157-63. [PubMed: 11301234]

8. De Jong R. Multiple bottlenecks in overlapping task performance. Journal of Experimental Psychology: Human Perception and Performance. 1993; 19:965-80. [PubMed: 8228846]

9. Deubel H, Schneider WX. Saccade target selection and object recognition: evidence for a common attentional mechanism. Vision Research. 1996; 36:1827-37. [PubMed: 8759451] 
10. Friston, KJ. Statistical parametric mapping: the analysis of functional brain images. London: Academic; 2007.

11. Friston, KJ.; Ashburner, J.; Kiebel, SJ.; Nichols, TE.; Penny, WD. Statistical parametric mapping: the analysis of functional brain images. New York: Academic Press; 2007.

12. Goldberg TE, Berman KF, Fleming K, Ostrem J, Van Horn JD, Esposito G, et al. Uncoupling cognitive workload and prefrontal cortical physiology: a PET rCBF study. NeuroImage. 1998; 7:296-303. [PubMed: 9626670]

13. Griew S. Set to respond and the effect of interrupting signals upon tracking performance. Journal of Experimental Psychology. 1959; 57:333-7. [PubMed: 13654645]

14. Grinband J, Wager TD, Lindquist M, Ferrera VP, Hirsch J. Detection of time-varying signals in event-related fMRI designs. NeuroImage. 2008; 43:509-20. [PubMed: 18775784]

15. Herath P, Klingberg T, Young J, Amunts K, Roland P. Neural correlates of dual task interference can be dissociated from those of divided attention: an fMRI study. Cerebral Cortex. 2001; 11:796805. [PubMed: 11532885]

16. Holmes A, Friston K. Generalisability, random effects and population inference. NeuroImage. 1998; 7:S754.

17. Holtzer R, Stern Y, Rakitin BC. Age-related differences in executive control of working memory. Memory \& Cognition. 2004; 32:1333-45.

18. Jiang Y. Resolving dual-task interference: an fMRI study. NeuroImage. 2004; 22:748-54. [PubMed: 15193603]

19. Johansen-Berg H, Rushworth MF, Bogdanovic MD, Kischka U, Wimalaratna S, Matthews PM. The role of ipsilateral premotor cortex in hand movement after stroke. Proceedings of the National Academy of Science of the United States of America. 2002; 99:14518-23.

20. Johnston JC, McCann RS. On the locus of dual-task interference: is there a bottleneck at the stimulus classification stage? Quarterly Journal of Experimental Psychology. 2006; 59:694-719.

21. Jolicoeur P. Restricted attentional capacity between sensory modalities. Psy-chonomics Bulletin and Review. 1999; 6:87-92.

22. Just MA, Carpenter PA, Keller TA, Emery L, Zajac H, Thulborn KR. Interdependence of nonoverlapping cortical systems in dual cognitive tasks. NeuroImage. 2001; 14:417-26. [PubMed: 11467915]

23. Just MA, Keller TA, Cynkar J. A decrease in brain activation associated with driving when listening to someone speak. Brain Research. 2008; 1205:70-80. [PubMed: 18353285]

24. Karlin L, Kestenbaum R. Effects of number of alternatives on the psychological refractory period. Quarterly Journal of Experimental Psychology. 1968; 20:167-78. [PubMed: 5653420]

25. Koch I. The role of crosstalk in dual-task performance: evidence from manipulating response-code overlap. Psychological Research. 2009; 73:417-24. [PubMed: 18443819]

26. Lancaster JL, Woldorff MG, Parsons LM, Liotti M, Freitas CS, Rainey L, et al. Automated Talairach atlas labels for functional brain mapping. Human Brain Mapping. 2000; 10:120-31. [PubMed: 10912591]

27. Liu CC, Doong JL, Hsu CC, Huang WS, Jeng MC. Evidence for the selective attention mechanism and dual-task interference. Applied Ergonomics. 2009; 40:341-7. [PubMed: 19166992]

28. Miller J, Ulrich R. Bimanual response grouping in dual-task paradigms. Quarter Journal of Experimental Psychology. 2008; 61:999-1019.

29. Miller JO, Low K. Motor processes in simple, go/no-go, and choice reaction time tasks: a psychophysiological analysis. Journal of Experimental Psychology: Human Perception and Performance. 2001; 27:266-89. [PubMed: 11318047]

30. Monty RA, Ruby WJ. Effects of added work load on compensatory tracking for maximum terrain following. Human Factors. 1965; 7:207-14. [PubMed: 5867015]

31. Netick A, Klapp ST. Hesitations in manual tracking: a single-channel limit in response programming. Journal of Experimental Psychology: Human Perception \& Performance. 1994; 20:766-82. [PubMed: 8083633]

32. Pashler H. Dissociations and dependencies between speed and accuracy: evidence for a twocomponent theory of divided attention in simple tasks. Cognitive Psychology. 1989; 21:469-514. 
33. Pashler H, Johnston JC. Chronometric evidence for central postponement in temporally overlapping tasks. The Quarterly Journal of Experimental Psychology A: Human Experimental Psychology. 1989; 41:19-45.

34. Pashler H. Dual-task interference in simple tasks: data and theory. Psychological Bulletin. 1994; 116:220-44. [PubMed: 7972591]

35. Poulton, EC. Tracking skill and manual control. New York: Academic Press; 1974.

36. Pruessmann KP, Weiger M, Scheidegger MB, Boesiger P. SENSE: sensitivity encoding for fast MRI. Magnetic Resonance in Medicine. 1999; 42:952-62. [PubMed: 10542355]

37. Rushworth MF, Johansen-Berg H, Gobel SM, Devlin JT. The left parietal and premotor cortices: motor attention and selection. NeuroImage. 2003; 20(Suppl 1):S89-100. [PubMed: 14597301]

38. Schluter ND, Krams M, Rushworth MF, Passingham RE. Cerebral dominance for action in the human brain: the selection of actions. Neuropsychologia. 2001; 39:105-13. [PubMed: 11163368]

39. Schubert T, Szameitat AJ. Functional neuroanatomy of interference in overlapping dual tasks: an fMRI study. Cognitive Brain Research. 2003; 17:733-46. [PubMed: 14561459]

40. Schubert T. The central attentional limitation and executive control. Frontiers in Bioscience. 2008; 13:3569-80. [PubMed: 18508456]

41. Stelzel C, Kraft A, Brandt SA, Schubert T. Dissociable neural effects of task order control and task set maintenance during dual-task processing. Journal of Cognitive Neuroscience. 2008; 20:61328. [PubMed: 18052784]

42. Sternberg S. The discovery of processing stages: extensions of Donders' method. Acta Psychologia. 1969; 30:276-315.

43. Strayer DL, Johnston WA. Driven to distraction: dual-Task studies of simulated driving and conversing on a cellular telephone. Psychological Science. 2001; 12:462-6. [PubMed: 11760132]

44. Szameitat AJ, Lepsien J, von Cramon DY, Sterr A, Schubert T. Task-order coordination in dualtask performance and the lateral prefrontal cortex: an event-related fMRI study. Psychological Research. 2006; 70:541-52. [PubMed: 16142491]

45. Telford CW. The refractory phase of voluntary and associative responses. Journal of Experimental Psychology. 1931; 14:1-36.

46. Tunik E, Houk JC, Grafton ST. Basal ganglia contribution to the initiation of corrective submovements. NeuroImage. 2009

47. van Mier H, Hulstijn W, Petersen SE. Changes in motor planning during the acquisition of movement patterns in a continuous task. Acta Psychologica (Amsterdam). 1993; 82:291-312.

48. Worsley KJ, Marrett S, Neelin P, Vandal AC, Friston KJ, Evans AC. A unified statistical approach for determining significant signals in images of cerebral activation. Human Brain Mapping. 1996; 4:58-73. [PubMed: 20408186]

49. Zarahn E. Testing for neural responses during temporal components of trials with BOLD fMRI. NeuroImage. 2000; 11:783-96. [PubMed: 10860802] 

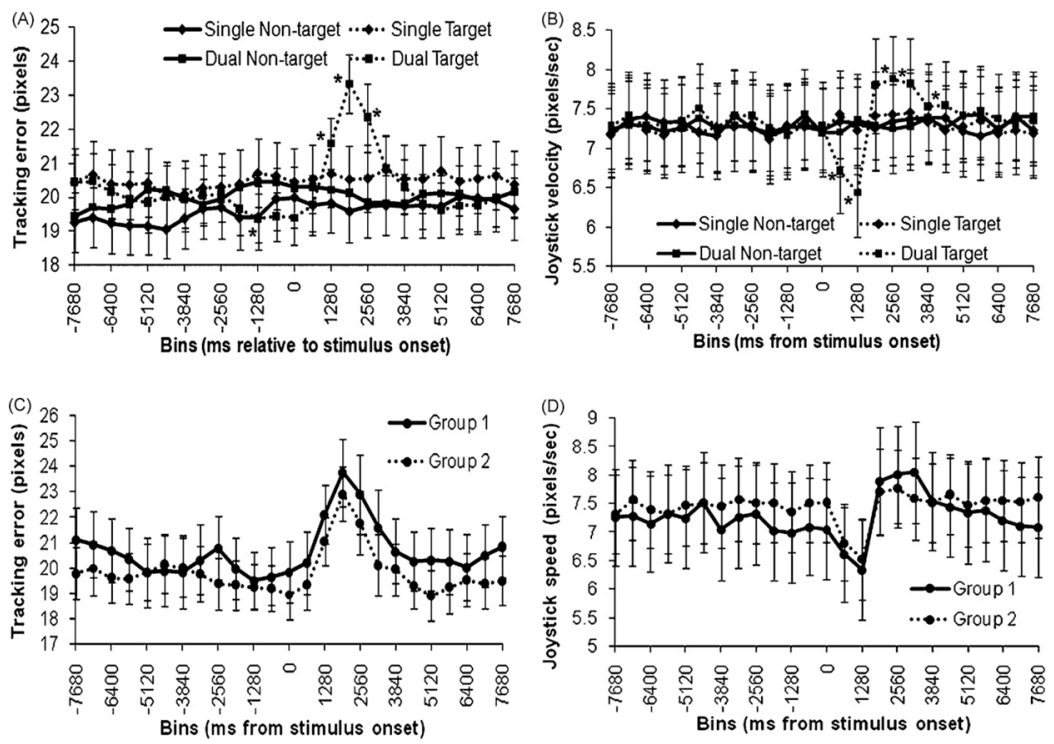

Fig. 1.

Error bars represent standard error. Top: means for binned (A) tracking error and (B) joystick speed time-locked to target (dotted line) and non-target (solid line) stimulus onset in single (diamond) and dual (square) task conditions. Asterisks mark the bins significantly different from the grand mean. Bottom: means for binned (A) tracking error and (B) joystick speed time-locked to target stimulus onset for Group 1 (solid line) and Group 2 (dotted line). 


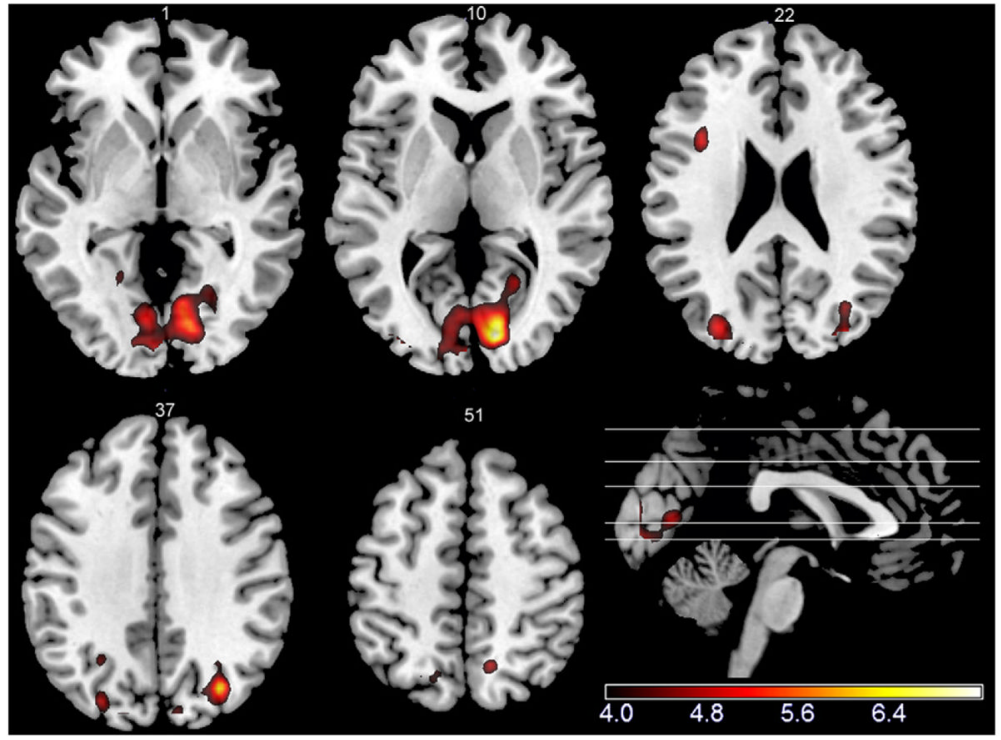

Fig. 2.

Results of the whole-brain random effects analysis for non-target stimuli with greater activation in the dual than in the single-task condition. Shown at the Bonferroni-corrected $t$ $>4.09$. Numbers above brain slices indicate the Talairach $z$ coordinates. 


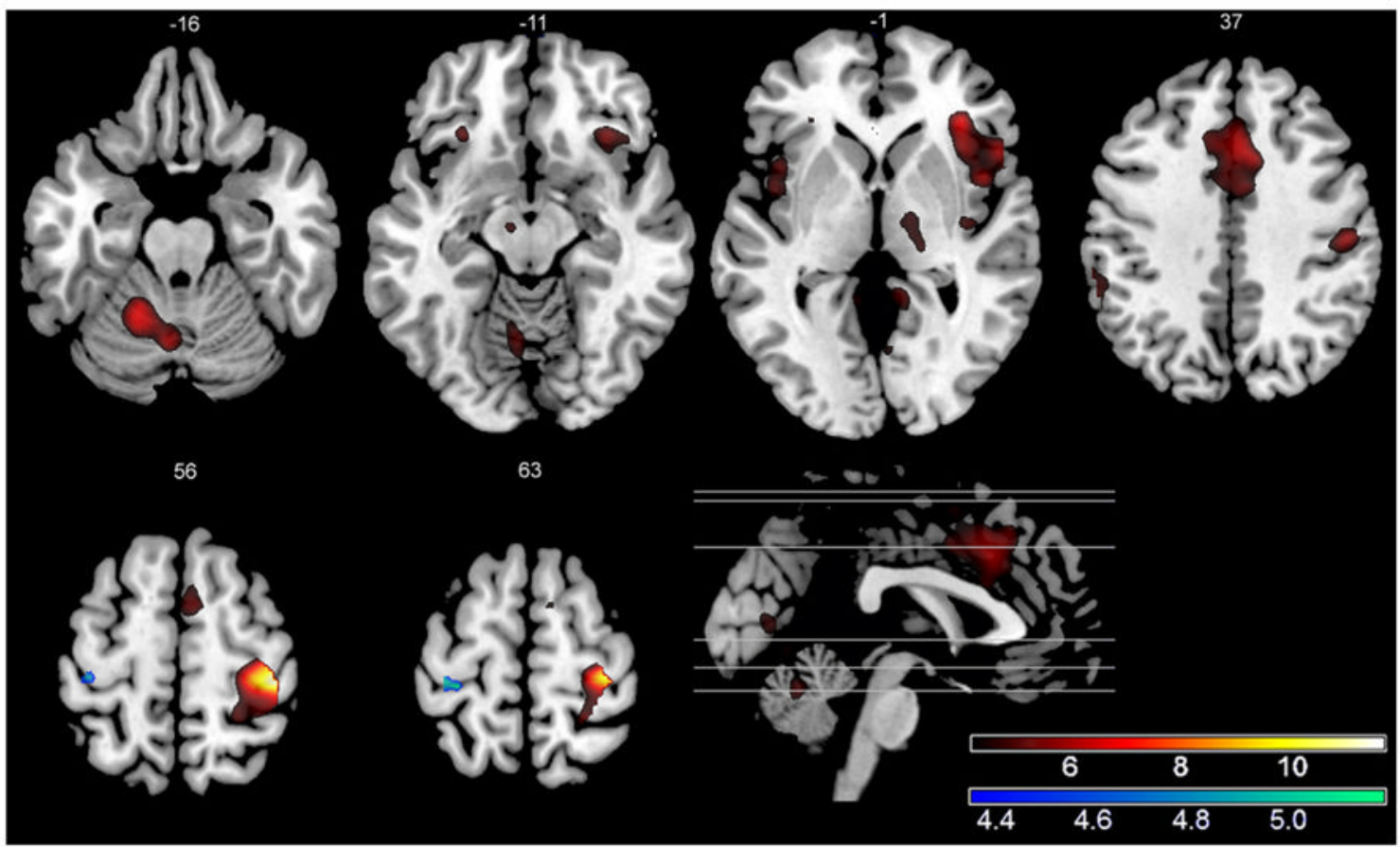

Fig. 3.

Results of the whole-brain random effects analysis for target vs. non-target stimuli in the dual-task condition. Shown at the Bonferroni-corrected $t>4.11$. Numbers above brain slices indicate the Talairach $z$ coordinates. Hot colors $=$ Target $>$ Non-target. Cool colors $=$ Nontarget $>$ Target. 


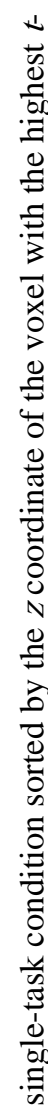




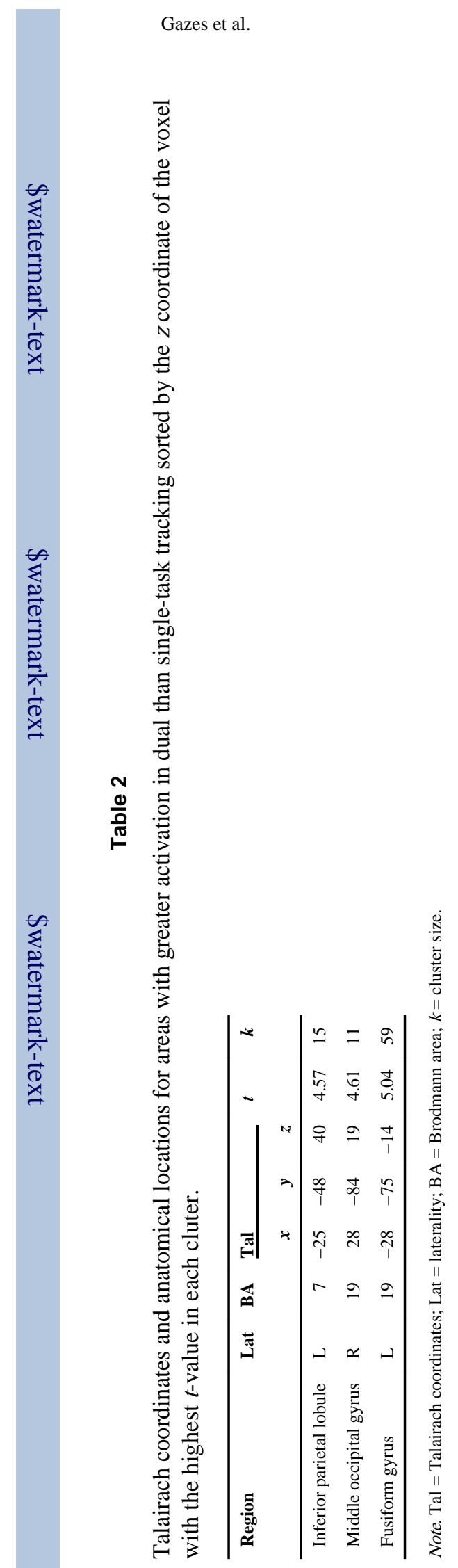

Behav Brain Res. Author manuscript; available in PMC 2012 December 27. 

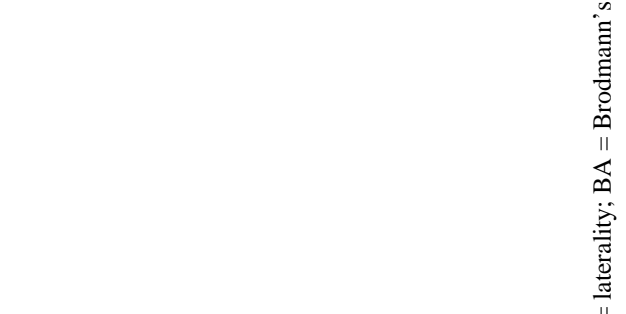

$-12 \cos 2$

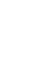




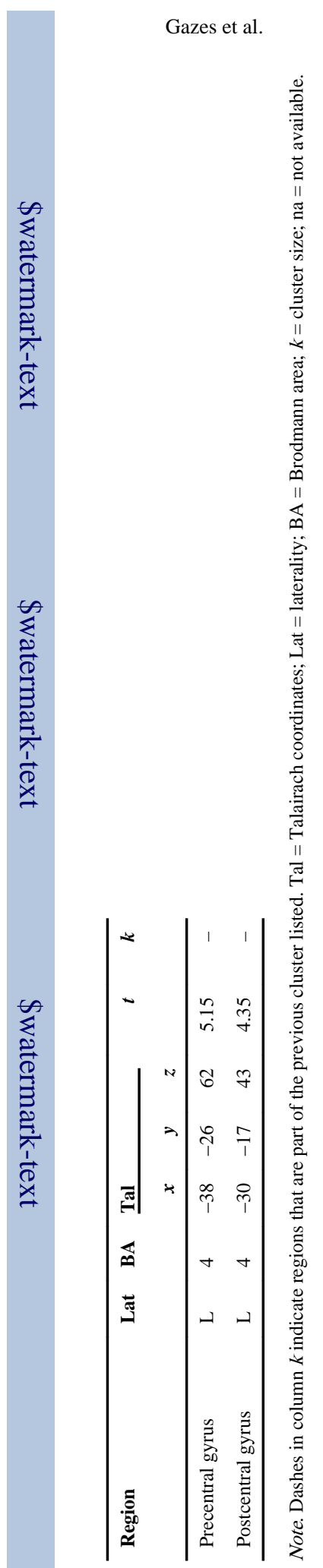

Page 24

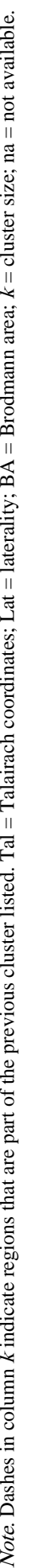


$\Xi$

先

$\stackrel{\overrightarrow{0}}{0}$

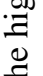

言

Е

$\stackrel{0}{\overparen{7}}$

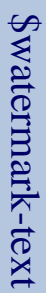

$=$
-0
0
0
0
0
00
0
3
0
0
0
0
0
0
0
5
0

官

尫

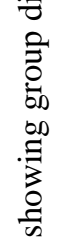

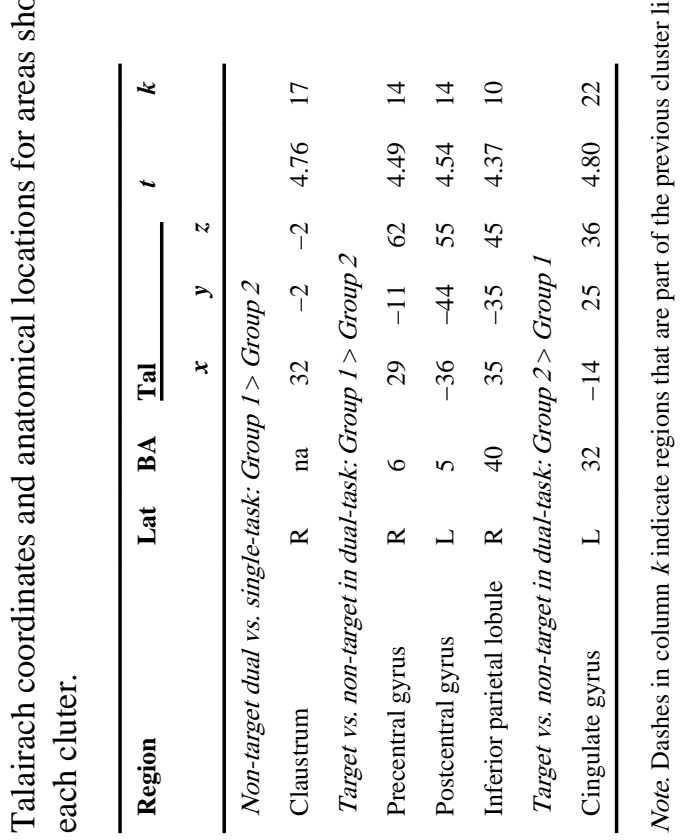

Behav Brain Res. Author manuscript; available in PMC 2012 December 27. 